\title{
Short-term resistance exercise inhibits neuroinflammation and attenuates neuropathological changes in 3xTg Alzheimer's disease mice
}

\author{
Yan Liu ${ }^{1,2}$, John Man Tak Chu ${ }^{1,2}$, Tim Yan ${ }^{2}$, Yan Zhang ${ }^{1,2}$, Ying Chen ${ }^{1,2}$, Raymond Chuen Chung Chang ${ }^{2,3^{*}}$ and \\ Gordon Tin Chun Wong ${ }^{1 *}$ (D)
}

\begin{abstract}
Background: Both human and animal studies have shown beneficial effects of physical exercise on brain health but most tend to be based on aerobic rather than resistance type regimes. Resistance exercise has the advantage of improving both muscular and cardiovascular function, both of which can benefit the frail and the elderly. However, the neuroprotective effects of resistance training in cognitive impairment are not well characterized.

Methods: We evaluated whether short-term resistant training could improve cognitive function and pathological changes in mice with pre-existing cognitive impairment. Nine-month-old 3xTg mouse underwent a resistance training protocol of climbing up a 1-m ladder with a progressively heavier weight loading.

Results: Compared with sedentary counterparts, resistance training improved cognitive performance and reduced neuropathological and neuroinflammatory changes in the frontal cortex and hippocampus of mice. In line with these results, inhibition of pro-inflammatory intracellular pathways was also demonstrated.

Conclusions: Short-term resistance training improved cognitive function in 3xTg mice, and conferred beneficial effects on neuroinflammation, amyloid and tau pathology, as well as synaptic plasticity. Resistance training may represent an alternative exercise strategy for delaying disease progression in Alzheimer's disease.
\end{abstract}

Keywords: Resistance exercise, Neuroinflammation, Alzheimer's disease, Tau, Amyloid, Cytokines, Synapse

\section{Background}

Alzheimer's disease (AD) is a chronic neurodegenerative disease that is characterized by a gradual loss of memory and cognitive function. Pathologically, accumulation of amyloid plaques and tau aggregation can be observed in several brain regions including the hippocampus and cortex [1]. Currently, there is no definitive therapeutic option for reversing $\mathrm{AD}$ progression, a process that involves neuroinflammation [2]. In postmortem brain of $\mathrm{AD}$ patients, upregulation of inflammatory cytokines

\footnotetext{
* Correspondence: rccchang@hku.hk; gordon@hku.hk

${ }^{2}$ Laboratory of Neurodegenerative Diseases, LKS Faculty of MedicineSchool of Biomedical Sciences, The University of Hong Kong, Hong Kong, SAR, China 'Department of Anaesthesiology, LKS Faculty of Medicine, The University of Hong Kong, Room K424, Queen Mary Hospital, Pokfulam, Hong Kong, SAR, China

Full list of author information is available at the end of the article
}

and activation of microglia can readily be seen $[3,4]$. Experimentally, increasing neuroinflammatory responses in the brain can exacerbate memory deficits [5], along with the exacerbation of amyloidosis and tauopathies [6]. Although therapeutic strategies targeting neuroinflammatory responses such as the use of non-steroid antiinflammatory drugs (NSAIDs) has been shown to be effective in ameliorating cognitive deficits [7], the merits of this approach remain controversial given the known adverse effects of chronic NSAIDs treatment [8].

Exercise training has been shown to be a powerful tool in combating neuroinflammation and cognitive dysfunction in $\mathrm{AD}$ [9] and represents an intervention that can elicit whole body responses in metabolism and immunity [10]. Exercise training can be divided into two major types: endurance-based and resistance-based training. 
Each type results in different "reprogramming" of the muscle fiber to adapt to different purposes [10]. Although major studies highlight the peripheral advantages of exercise training, mounting evidence have also demonstrated beneficial effects in the central nervous system (CNS). Physical training preserves cognitive function in both human and rodents [11]. Moderate exercise training at different stages of life is associated with a reduced risk of developing cognitive impairment in the later life [12]. Cognitive performance of $\mathrm{AD}$ transgenic mice with excess $A \beta$ production improved after 5 months of treadmill training [13]. An anti-neuroinflammatory effect has been proposed to underlie the neuroprotective mechanism of exercise. Exercise training can significantly reduce inflammatory cytokines and active microglia in the brains of aged or AD animals [14]. Nevertheless, most studies focused on endurance-based exercise training, while the neuroprotective effects of resistance-based training remain to be elucidated. In particular, the impact of resistance training on neuroinflammation and cognitive dysfunction deserve further clarification. We hypothesized that resistance training can suppress neuroinflammation and improve cognitive deficits. We will demonstrate our findings in an experimental AD animal model using transgenic mice with over-expression of amyloid peptide precursor, tau, and presenilin-1 (3xTg). This study aims to demonstrate the therapeutic potential of a short-term resistance training regimen especially in terms of attenuating neuroinflammation, improving synaptic function, and minimizing neuropathological changes in the brain.

\section{Methods}

\section{Materials and methods}

Animals

Nine-month-old male transgenic AD mice (B6; 129Psen $^{1 t m 1 M p m} \mathrm{Tg}$ (APPSwe, tauP301L) 1L fa/MmJax) were obtained from the Jackson Laboratory and were held in Laboratory Animal Unit (LAU) of The University of Hong Kong, which is fully accredited by the Association for Assessment and Accreditation of Laboratory Animal Care International; 9-month-old age-matched nontransgenic mice were used as healthy controls. Mice were housed in a temperature-controlled room at 20 $22{ }^{\circ} \mathrm{C}$, humidity of $50 \pm 10 \%$ and were kept on a $12 / 12 \mathrm{~h}$ light/dark cycle. All mice had access to food and water ad libitum. Mice handling and all other procedures were conducted in accordance with the National Institutes of Health guide for the care and use of laboratory animals and the Animals (Control of Experiments) Ordinance, Hong Kong, China. The use of animals was approved by the Department of Health, Hong Kong and the Committee on the Use of Live Animals in Teaching and Research, The University of Hong Kong. All efforts were made to minimize animal numbers and suffering. For transgenic mice, the experiment was conducted in two batches: each batch contains a total of 15 mice with seven in sedentary group and eight in training group. Sixteen non-transgenic mice were randomly assign to sedentary and exercise group with eight mice in each group.

\section{Resistance training protocol}

Mice were trained following a previously published protocol that uses a 1-m ladder [15] as shown in Fig. 1a. Familiarization with the exercise apparatus took place over 1 week by allowing the animals to climb up the ladder with no added resistance. Training then began on alternate days for the following 4 weeks. If the resistance training and behavior tests were at the same day, then the resistance training would be performed $2 \mathrm{~h}$ after the behavior test. For each training session, the mice were motivated to climb up the ladder to a total of 15 times, with progressively heavier weights attached to their tails and 2-min rest in between each climb. The weights that were loaded were equivalent to $15 \%, 30 \%, 50 \%$, and $75 \%$ of their body weight in weeks $1,2,3$, and 4 respectively. The intensity was carefully adjusted based on their individual difference during each exercise session.

Excessive stress was avoided during this training protocol. All the mice were trained to climb up the ladder spontaneously, only motivated with a gentle touch to the tail when they stopped in the middle of the ladder (see Additional file 1). For mice that showed symptoms of burnout (for example a failure to return to their baseline breathing pattern within 2 min or a refusal to climb), they were allowed to take extra rest time. If they still refused to climb up, the weight attached to their tail will be progressively reduced by $5 \mathrm{~g}$ until they resume. The reduced weight will be added back in the following trials.

\section{Open field test}

The open field test is used to assess locomotor activity, anxiety, and depression in rodents. Mice were brought into the dim light behavior assessment room and were allowed to habituate there for $30 \mathrm{~min}$. Each mouse was then gently placed in the middle of a $40 \mathrm{~cm}^{2}$ enclosed gridded arena and allowed to freely explore the area and $10 \mathrm{~min}$ of this spontaneous activity was video recorded. A video tracking software (SMART 3.0, Panlab SL) were used for data analysis. Brefily, the arena were equally divided into nine zones (as showed in Fig. 1g), a central area (zone 5) of $13.3 \times 13.3 \mathrm{~cm}^{2}$ was demarcated and the total exploration time in this area was calculated as the parameter for anxiety/depression, and the total transition during different rooms, total distance traveled, and mean speed were documented as indicators of locomotor activity. 


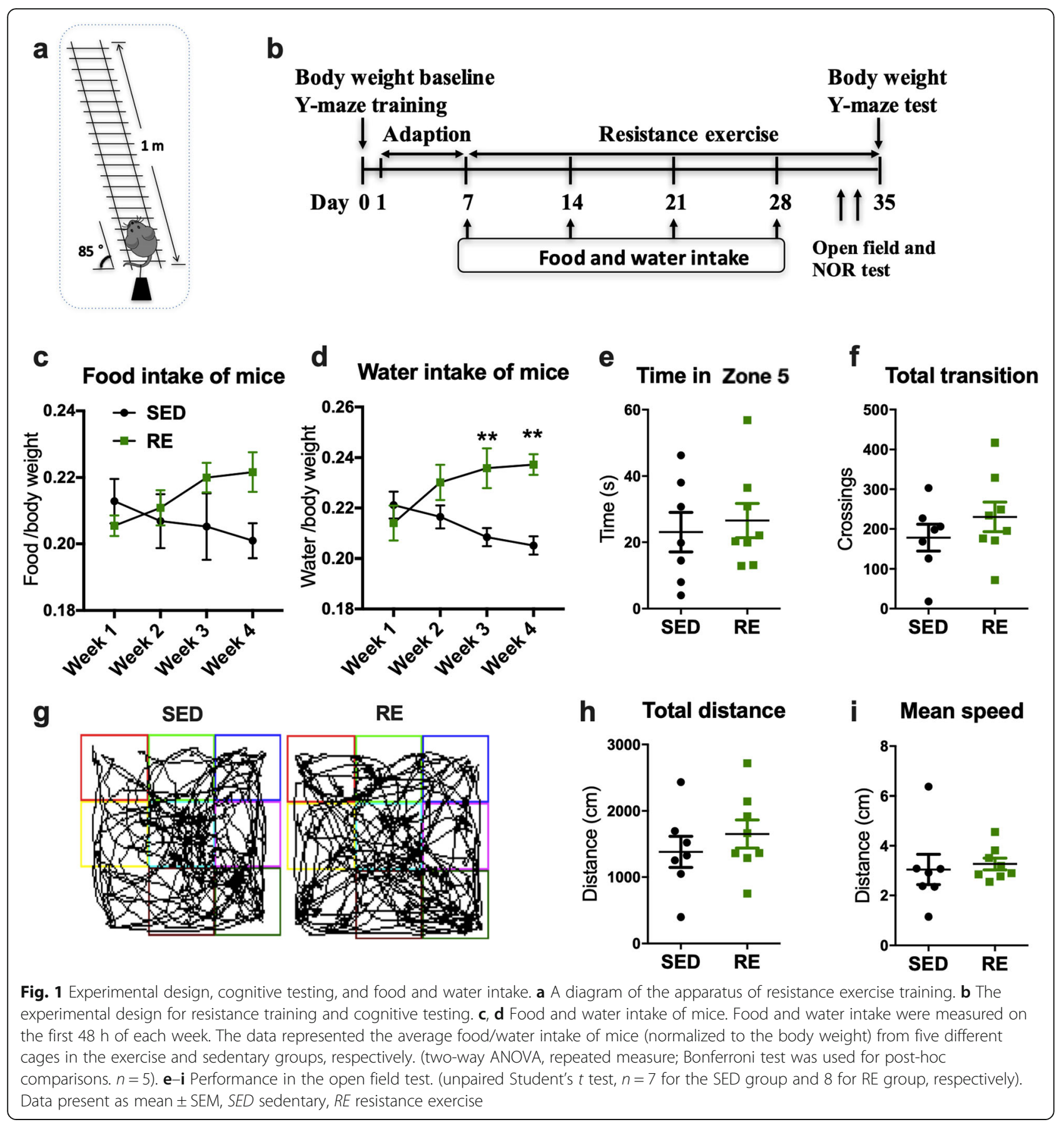

Novel object recognition test

The novel object recognition (NOR) task was performed to evaluate the animals' ability to recognize a novel object in a controlled environment. After $24 \mathrm{~h}$ of habituation in the open field arena in the absence of any objects, two identical sample objects $(\mathrm{A}+\mathrm{A})$ were placed at opposite but symmetrical corners of the arena. The mice were then put into the arena and allowed to explore the two objects freely for $10 \mathrm{~min}$. Six hours later, one of the objects (A) was replaced by a novel object
(B); the mice were placed back to the same arena with two objects $(A+B)$. The location of the novel versus familiar object was alternated within each batch of test such that the number of times the familiar and novel object was placed at a particular corner was similar. The mice were allowed to explore the objects for $10 \mathrm{~min}$ and the interaction of the mouse with the objects was video recorded. An interaction was defined by the nose of the mouse pointing to the object within a distance of $2 \mathrm{~cm}$. The discriminating index is the ratio of the time spent 
exploring the two objects (B/A) and this index was used as a measure of recognition memory.

\section{Y-maze test}

The Y-maze test was used to examine hippocampal dependent memory function according to our previous protocol [16]. Briefly, the mice were placed in a Y-shape apparatus of which two black compartments are able to deliver electric shocks $(2 \mathrm{~Hz}, 10 \mathrm{~s}, 40 \pm 5 \mathrm{~V})$. After being placed into the maze, electric shocks were delivered to motivate the mice to find and enter the shock-free illuminated compartment and the test is completed when it stays there for $30 \mathrm{~s}$. Each mouse was trained before undergoing the exercise training protocol and the training was deemed successful if they made nine consecutive correct choices. At the end of the training period, the Ymaze test was again conducted and the number of errors and the escape latency to enter the shock-free compartment were recorded.

\section{mRNA extraction and real time PCR}

The mice were sacrificed by $\mathrm{CO}_{2}$ asphyxiation and were perfused via a transcardial approach with $15 \mathrm{ml}$ of cold $0.9 \%$ saline to rinse away residual blood. The brains were removed and the left hemispheres, together with the gastrocnemius muscle, were immediately immersed in precooled $4 \%$ paraformaldehyde in phosphate buffer and kept at $4{ }^{\circ} \mathrm{C}$ for further studies. The frontal cortices and hippocampi were dissected from the right hemispheres. The mRNA of the livers and brains was isolated by RNAiso plus (Takara, Japan) as previously described [16]. Isolated mRNA was dissolved in DEPC water and $1 \mu \mathrm{g}$ of mRNA was converted to complementary DNA sequence by reversed transcription using cDNA synthesis kit according to manufactures' protocol (Takara, Japan). cDNA expression of different inflammatory cytokines and exercise-induced factors were assessed by real-time PCR with the following primers respectively: (1) IL-1ß, forward: CCTCCTTGCCTCTG ATGG, reverse: AGTGCTGCCTAATGTCCC; (2) IL6, forward: TTCACAAGTCCGGAGAGGAG, reverse: TCCACGATTTCCCAGAGAAC; (3) TNF- $\alpha$, forward: CCCCAGTCTGTATCCTTCT, reverse: ACTGTCCC AGCATCTTGT; (4) IL-10, forward: CCAAGCCTTA TCGGAAATGA, reverse: TTCTCACCCAGGGAATTCAA; (5) FGF-21, forward: AGATCAGGGAGGATGG AACA, reverse: TCAAAGTGAGGCGATCCATA; 6) PGC1- $\alpha$ : forward: AACGATGACCCTCCTCACAC, reverse: TCTGGGGTCAGAGGAAGAGA; (7) glyceraldehyde 3-phosphate dehydrogenase (GAPDH), forward: ATTCAACGGCACAGTCAA, reverse: CTCG CTCCTGGAAGATGG.

\section{Milliplex cytokine assays}

Protein levels of IL-1 $\beta$, IL-6, IL-10, MCP-1, MIP-2, and TNF- $\alpha$ in the serum were measured using a customized Milliplex Mouse Cytokine Immunoassay Kit (Millipore, 2620525) with Analyzer 3.1 Luminex 200 machine (Millipore, USA). Data were analyzed using corresponding software according to the manufacturer's instructions.

\section{Western blot}

After being sacrificed by $\mathrm{CO}_{2}$ asphyxiation, the frontal cortical and hippocampal tissues dissected from the left hemispheres of each mouse were homogenized in RIPA lysis buffer (Cellsignal, Danvers, MA) supplemented with protease and phosphatase inhibitors (Roche, Berlin, German). Tissues from the right hemispheres were used to evaluate the expression levels of synaptic proteins, then the synaptosomal and cytosolic fractions were freshly prepared by using Syn-PER ${ }^{\mathrm{TM}}$ Synaptic Protein Extraction Reagent (Thermo Fisher Scientific, USA) plus protease and phosphatase inhibitors (Roche, Berlin, German), following by the manufacturer's instructions. The levels of intracellular signal proteins and synaptic proteins expressed were evaluated by Western blot analysis.

Proteins were resolved by SDS-PAGE gel and transferred to PVDF membrane (Bio-Rad, USA). After blocking with $5 \%$ non-fat milk in TBST $(0.1 \%$ tween 20 in TBS), membranes were probed with different primary antibodies (Synapsin I, Thermo Fisher Scientific, MA; Synaptotagmin 1, Synaptobrevin 1 were from Synaptic Systems, Germany; Pan-tau was from DAKO, Japan; ptau S396, p-tau S404 were from Thermo Fisher Scientific, MA; AT180 was from Innogenetics, Belgium; GADPH was from Sigma-Aldrich, UK; PSD 95, c-Jun Nterminal kinases (JNK), p-JNK, extracellular signalregulated kinases (ERK), p-ERK, Akt, p-Akt, Bax, and BCL-2 were from Cellsignal, MA) overnight at $4{ }^{\circ} \mathrm{C}$, followed by respective HRP-conjugated secondary antibodies for $1 \mathrm{~h}$. Protein bands were visualized by enhanced chemiluminescence (ECL) reagents (Advansta, USA) and signals were captured by ChemiDoc ${ }^{\mathrm{Tx}}$ Touch Imaging System (Bio-Rad, USA). The intensities of protein bands were quantified by Image $\mathrm{Lab}^{\text {Ta }}$ Touch Software Version 1.2 (provided by Bio-Rad, USA).

\section{Immunofluorescent staining}

The brains were immersed in $4 \%$ paraformaldehyde overnight at $4{ }^{\circ} \mathrm{C}$, then immersed in $20 \%$ sucrose following by $30 \%$ sucrose for further cryoprotection. The brains were cut into $20 \mu \mathrm{m}$ thickness frozen crosssections for immunofluorescent (IF) staining of Iba-1 and immunohistochemical (IHC) staining; to perform morphology analysis for astrocyte, sections in $30 \mu \mathrm{m}$ 
thickness were used for GFAP staining. For IF staining, sections were immersed with 5\% BSA (Thermo Fisher Scientific, MA) for $60 \mathrm{~min}$ at room temperature to block non-specific antigens, and then were incubated with anti-Iba-1 monoclonal antibody (1:300, Wako, Japan) or anti-GFAP antibody (1:500, Sigma) overnight at $4{ }^{\circ} \mathrm{C}$. After the sections were rinsed three times in PBS, they were incubated with fluorochrome-conjugated secondary antibodies (anti-rabbit, Alexa-fluor 488 for Iba-1, or anti-mouse, Alexa-fluor 568 for GFAP; Thermo Fisher, USA) in a cassette at room temperature for $1 \mathrm{~h}$. Slight agitation was applied throughout and this was followed by incubation with $3 \mathrm{mM}$ DAPI (Thermo Fisher, USA) for $15 \mathrm{~min}$ at room temperature. The slices were then mounted using mounting medium (DAKO, Japan). LSM700 and LSM780 confocal microscopy (Carl Zeiss, Germany) were used to examine the IBA-1 and GFAP signals, respectively, and the z-stacked signals were collected. Quantification of the images was performed using ImageJ software (National Institute of Health, USA). The morphological analysis of astrocytes was performed with the Simple Neurite Tracer plugin of ImageJ [17]. Astrocytes meeting the following criteria were included for morphology analysis (see Fig. 6d for representative examples): GFAP-stained bushy shape with a single DAPIstained nucleus and relatively intact with no more than two truncated primary processes.

\section{Immunohistochemistry staining}

Brain slices from four mice in each group were stained simultaneously with purified anti- $\beta$-amyloid antibody (clone 4G8, was from Biolegend, USA). Briefly, following 20 min of antigen retrieval using $88 \%$ formic acid at room temperature, the slices were incubated with $1 \mu \mathrm{g} /$ $\mathrm{ml}$ of the primary antibody for $60 \mathrm{~min}$ at room temperature. This was followed by hematoxylin counterstaining and DAB (vector laboratories, USA) was used as chromogen for detection. The pictures of $A \beta$ staining were captured with a Leica microscope (Leica CTR 5000; Germany). Quantification of the $A \beta$ deposits was performed using ImageJ software.

\section{Statistical analysis}

Statistical analyses were performed using Prism 7.0 (Graphpad Software, USA). All data were displayed as mean \pm SEM. Body weights were analyzed using paired Student's $t$ test (compared to baseline). The unpaired Student's $t$ test was performed for behavioral tests, Western immunoblots, real-time PCR and IF staining, as well as IHC staining (compared to sedentary mice). The food and water intake during resistance training and the process complexity of astrocytes were analyzed using two-way ANOVA (repeated measure, post-hoc test:
Bonferroni test). The accepted level of significance in all cases was $p<0.05$.

\section{Results}

Resistance exercise training did not induce stress in $3 \times \mathrm{Tg}$ mice

Stressed animals often display abnormal patterns of eating, drinking, and signs of anxiety. To determine whether the animals were unduly stressed by undergoing resistance training, we carefully monitored the food and water intake of mice, which were normalized to their body weight. Food intake was not affected by resistance exercise (Fig. 1c), but mice in exercise group consumed more water compared with sedentary mice from the third week onwards (Fig. 1d. Week 3: $+0.02737, p=$ 0.0063 ; week $4:+0.03214, p=0.0012$ ). In addition, there was no difference in zone transition, central area duration time, total distance traveled, and mean movement speed in the open field test (Fig. 1e-i), suggesting that resistance protocol did not induce locomotor deficit or anxiety/depression in $3 x \mathrm{Tg}$ mice. Similarly, this training protocol did not induced locomotor deficit or anxiety/ depression in the wild-type mice (Additional file 2a-d).

\section{Resistance exercise training prevented body weight gain and preserved cognitive function in $3 \times \mathrm{Tg}$ mice}

Weight gain during adulthood may be associated with significantly increased risk of major chronic diseases and decreased odds of healthy aging [18]. Weight control is a well-known effect of exercise and the body weight of the mice was monitored throughout the experimental period (outlined in Fig. 1b). After 5 weeks, the mice in the sedentary group had increases in their body weight compared to their baselines $(2.286 \pm 0.5286 \mathrm{~g}, p=0.0008$; Fig. 2a). In contrast, the body weight of mice in the exercise group was significantly decreased compared to their baselines $(-2.813 \pm 0.5494 \mathrm{~g}, p<0.0001$; Fig. $2 \mathrm{a})$, in the absence of any decrease in food or water intake or any demonstrable stress (Fig. 1c-i). Similar changes in body weight were seen in wild-type mice (SED: $+2.25 \pm$ $0.7734, \quad p=0.0227 ; \quad$ RE: $\quad-3.25 \pm 0.5901, \quad p=0.0009$; Additional file 2e). Our results indicated that short-term resistance training reduced the body weight of middleaged mice compared with sedentary mice. In 3xTg mice, resistance training increased recognition index in the NOR test $(0.3315 \pm 0.1526, p=0.0384$, Fig. $2 b)$, as well as reduced the escape latency $(-1.504 \pm 0.519, p=0.0072$, Fig. 2c) and the number of errors $(-1.821 \pm 0.4174, p=$ 0.0002 , Fig. $2 \mathrm{~d}$ ) in the Y-maze test. However, resistance exercise did not induce any change in the cognitive function of wild-type mice using NOR and Y maze test (Additional file 2f-h). 


\section{a}

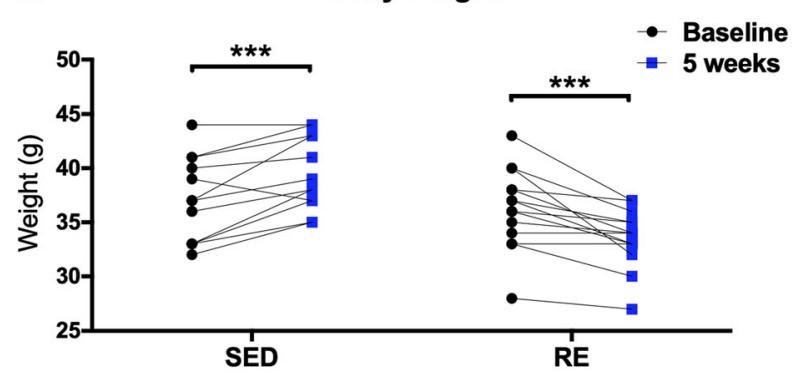

b NOR Test

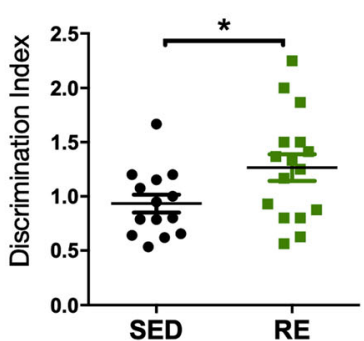

C Errors in Y-maze test

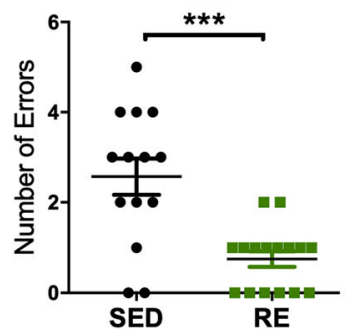

d Latency in Y-maze test

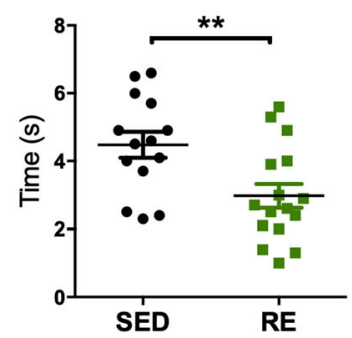

Fig. 2 Effects of resistance exercise on body weight and cognitive performance. a Body weight of mice. (paired Student's $t$ test, $n=7$ for SED group and 8 for RE group, respectively; compared to baseline). b Performance in the NOR test as assessed by the discrimination index. (unpaired Student's $t$ test, $n=14$ for SED group and 16 for RE group, respectively). c, $\mathbf{d}$ Y-maze training and test were performed before and at the end resistance exercise training respectively. Cognitive performance was assessed by the number of error and escape latency. (unpaired Student's $t$ test, $n=14$ for SED group and 16 for RE group, respectively). ${ }^{*} p<0.05,{ }^{* *} p<0.01,{ }^{* *} p<0.001$, data present as mean \pm SEM. SED sedentary, RE resistance exercise

Resistance exercise training increased the expression of synaptic proteins in $3 \times \mathrm{Tg}$ mice

Synaptic dysfunction and loss of synaptic markers are early changes seen in $\mathrm{AD}[6]$. Therefore, we evaluated the expression of several synaptic markers in frontal cortices and hippocampi of $3 \times \mathrm{Tg}$ mice, as these two brain regions are particularly vulnerable to neurodegenerative diseases and AD-related neuropathological [19]. Synapsin I, synaptotagmin 1, and synaptobrevin 1 are important presynaptic vesicle proteins, while PSD 95 is an important postsynaptic synaptic structure protein. We found an increase of synaptotagmin 1 from the synaptosome fraction of the frontal cortex of the active compared to the sedentary mice $(0.5964 \pm 0.2525, p=0.0458$; Fig. 3b). Similarly, the expression of synaptotagmin 1 and synaptobrevin 1 were increased in the synaptosome fraction of the hippocampi from the exercise group (synaptotagmin 1: $0.3662 \pm 0.1037, p=0.0064$; synaptobrevin 1: $0.401 \pm 0.1129, p=0.0062$; Fig. $3 c$ ). No difference was found in the level of synapsin I or PSD 95. This suggests that resistance exercise enhances presynaptic vesicular proteins, rather than postsynaptic structural proteins.
Resistance exercise training reduced amyloid deposits in the brain

Amyloid plaques and hyperphosphorylated tau, pathological hallmarks of $\mathrm{AD}$, can readily be seen in various brain regions of $3 x \mathrm{Tg}$ mice. We assessed the effect of resistance exercise on the degree of amyloid deposits seen in the frontal cortex (Fig. 3a) and hippocampus (Fig. 4c). The number of amyloid plaques were significantly reduced by resistance exercise in both the frontal cortex (-11.6 $\pm 3.326, p=0.0082$; Fig. $3 \mathrm{~b})$ and hippocampus $(-5.2 \pm 1.497, p=0.0084$; Fig. $4 d)$.

\section{Resistance exercise training reduced total and} hyperphosphorylated tau in the brain

We further evaluated the effect of resistance exercise on tau phosphorylation (AT180, p-tau S396, p-tau S404) and total tau using Western blot. The levels of total tau $(-0.3168 \pm 0.1142, p=0.0197$; Fig. $5 b)$ and phosphorylation of tau-AT180 ( $-0.5048 \pm 0.1358, p=0.004$; Fig. $5 \mathrm{~b})$ were significantly decreased in the frontal cortex; the level of phosphorylation of tau-AT180 (-0.5279 \pm 0.1978, $p=0.0236$, Fig. 5d) was also decreased in hippocampus in the exercise group. No significant changes were found for p-tau 396/404. 
a

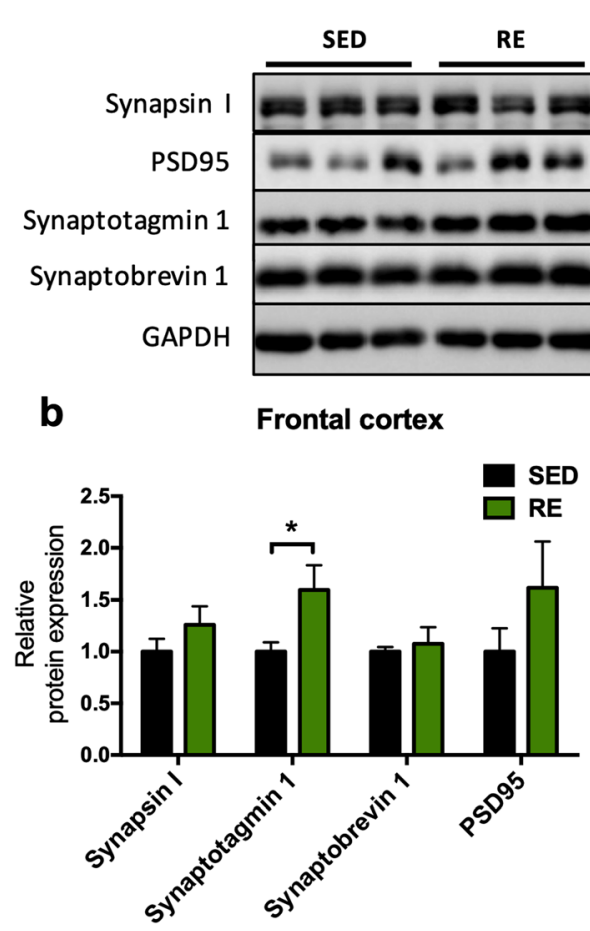

Hippocampus

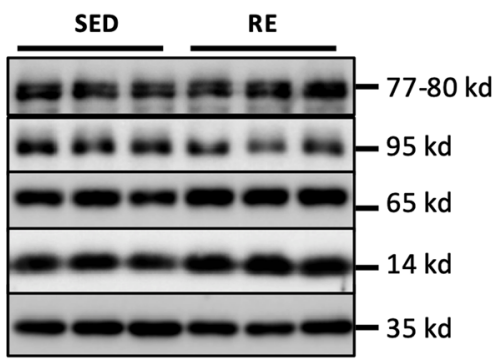

C

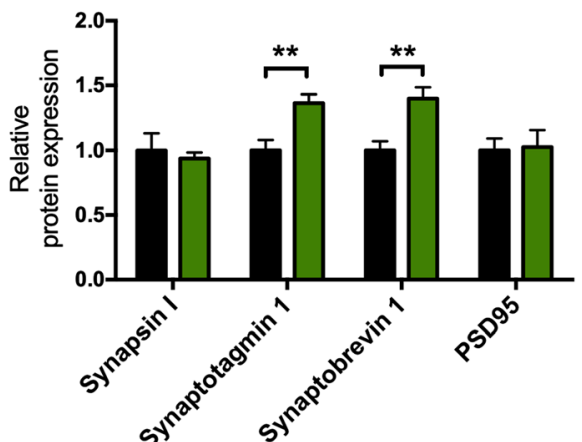

Fig. 3 Effects of resistance exercise on expression of synaptic proteins. a Representative blots of Synapsin I, PSD95, Synaptotagmin 1, and Synaptobrevin 1 for frontal cortex (left) and hippocampus (right). b, c The analysis of protein expression in the frontal cortex and hippocampus. Band intensity was normalized to that of GAPDH. (unpaired Student's $t$ tests, compared to sedentary mice. $n=5$ for SED group and $n=6$ for RE group, respectively. ${ }^{*} p<0.05,{ }^{* *} p<0.01$, data present as mean \pm SEM). SED sedentary, RE resistance exercise

a

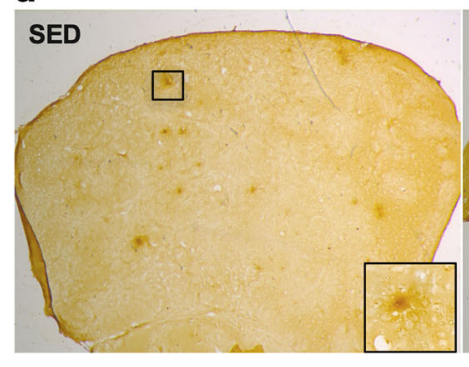

C

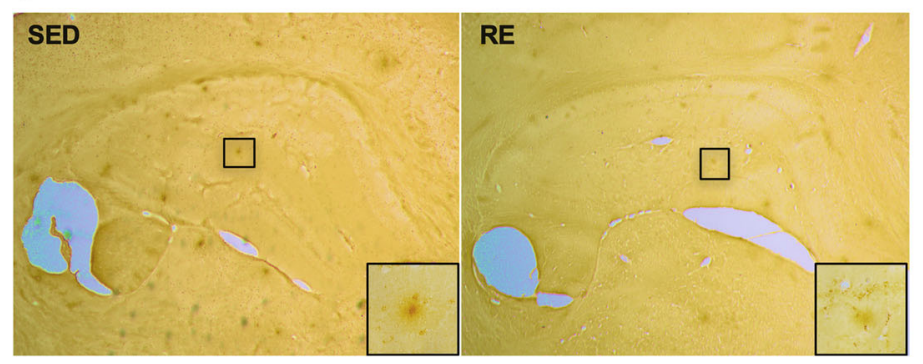

b

RE

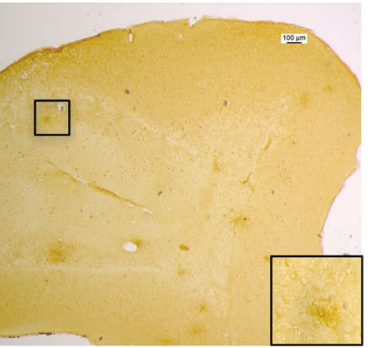

d

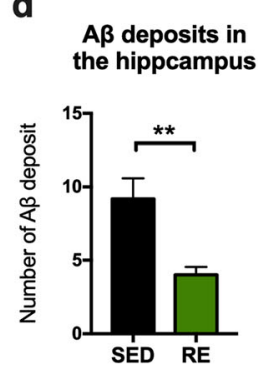

Fig. 4 Immunochemical staining of amyloid plaques. a, c Representative images of of amyloid plaques with a plaque-specific antibody (4G8) in the frontal cortex and the hippocampus. Zoomed-in image demonstrating typical morphology of amyloid deposit were presented in the bottom right corner of each image. Scale bar: $100 \mu \mathrm{m}$. b. d The number of amyloid plaques was analyzed using ImageJ. (unpaired Student's $t$ tests, compared to sedentary mice, ${ }^{*} p<0.05,{ }^{* *} p<0.01 . n=5$, data present as mean \pm SEM). SED sedentary, RE resistance exercise 

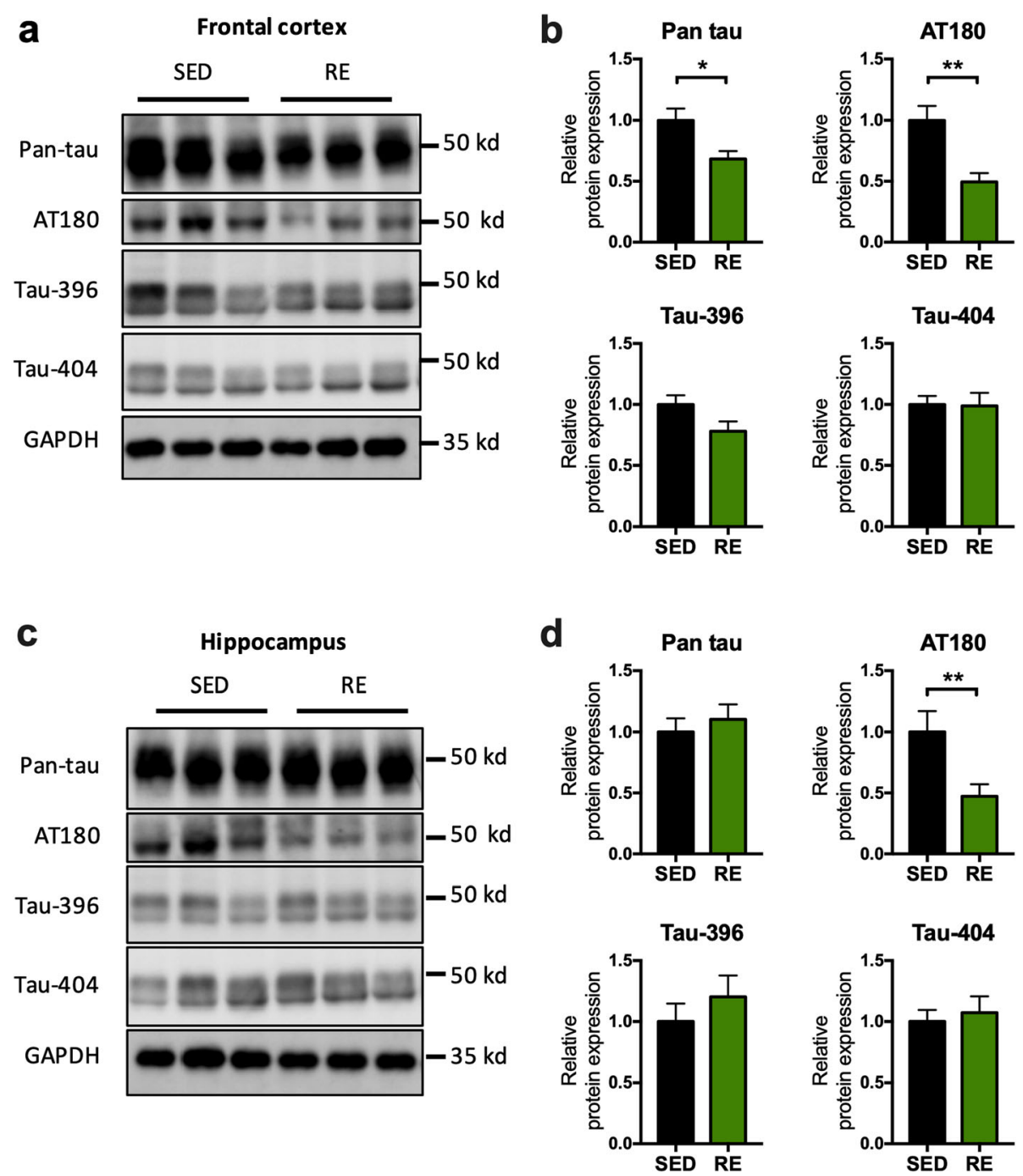

Fig. 5 Effects of resistance exercise on tau phosphorylation. a, c Representative blots of tau protein in the brain. $\mathbf{b}$, $\mathbf{d}$ The analysis of protein expression with the band intensity normalized to that of GAPDH. AT180 was the antibody used to detect tau phosphorylated at residue Thr 231. (unpaired Student's $t$ tests, compared to sedentary mice, ${ }^{*} p<0.05,{ }^{* *} p<0.01 . n=6$, data present as mean \pm SEM). SED sedentary, RE resistance exercise

Resistance exercise training reduced the activation of microglia and astrocytes in the brain

After resistance training, the number of Iba- $1^{+}$microglia were significantly decreased in the frontal cortex and the DG region of hippocampus compared to those from sedentary mice (frontal cortex: $-7 \pm 2.195, p=0.0128$; DG: $-8.4 \pm 1.778, p=0.0015$; Fig. $6 \mathrm{~b})$ with no significant difference found in other hippocampal regions (CA1: $6.8 \pm 5.312, p=0.2364 ;$ CA3: $-6.2 \pm 3.072, p=0.0783$; Fig. 6b). We further analyzed the cell body size of microglia and demonstrated smaller cell body in @the frontal cortex, DG, and CA3 region following resistance exercise (frontal cortex: $-0.1715 \pm 0.05951, p=0.0042$; CA1: -
$0.1214 \pm 0.07045, p=0.0861 ; \quad C A 3:-0.1549 \pm 0.07683$; $p=0.045$; DG: $-0.251 \pm 0.06598, p=0.0002$; Fig. $6 \mathrm{c}$ ).

Reactive astrogliosis in the hippocampus was assessed by GFAP staining. After resistance exercise, the fluorescence intensity of GFAP was significantly decreased in the DG region $(-0.522 \pm 0.1923, p=0.0349$; Fig. 6e) Further morphological analysis of the astrocytes in the DG region revealed that resistance exercise reduced the number of primary processes $(-2.875 \pm 0.7675, p=$ 0.0008; Fig. 6g), volume ( $54.8 \pm 19.28, p=0.008$; Fig. 6i), and process complexity within a radius of 4 to $13 \mu \mathrm{m}$ from the soma (radius $=4 \mu \mathrm{m}: 2.563 \pm 0.4609, p<0.0001$; radius $=7 \mu \mathrm{m}: \quad-1.938 \pm 0.4609, \quad p=0.0004 ;$ radius $=$ 
a
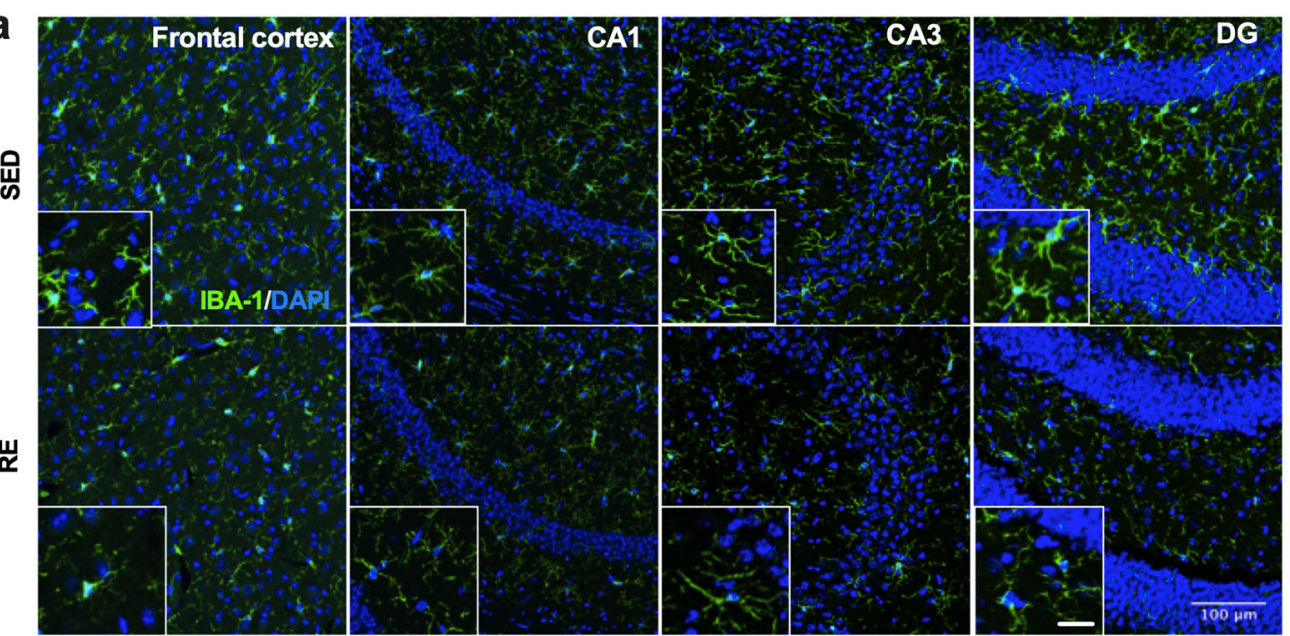

b
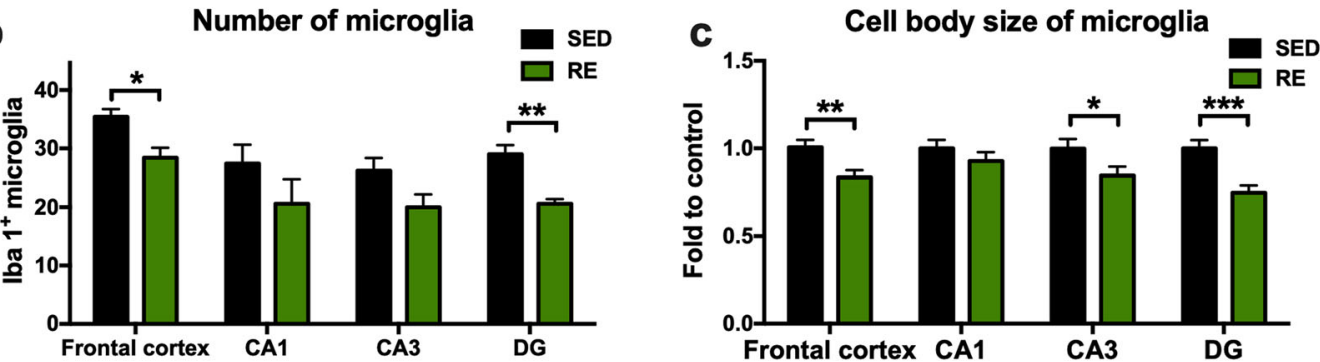

d
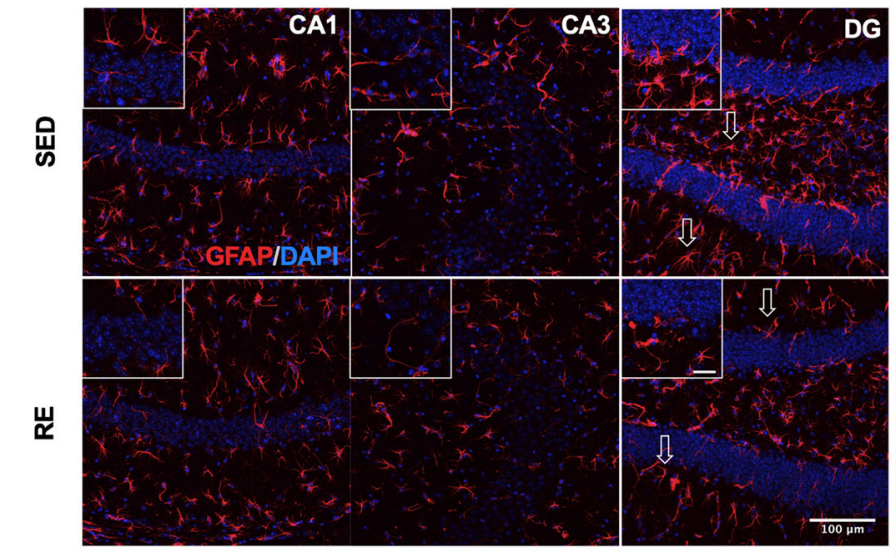

C Fluorescene intensity of GFAP
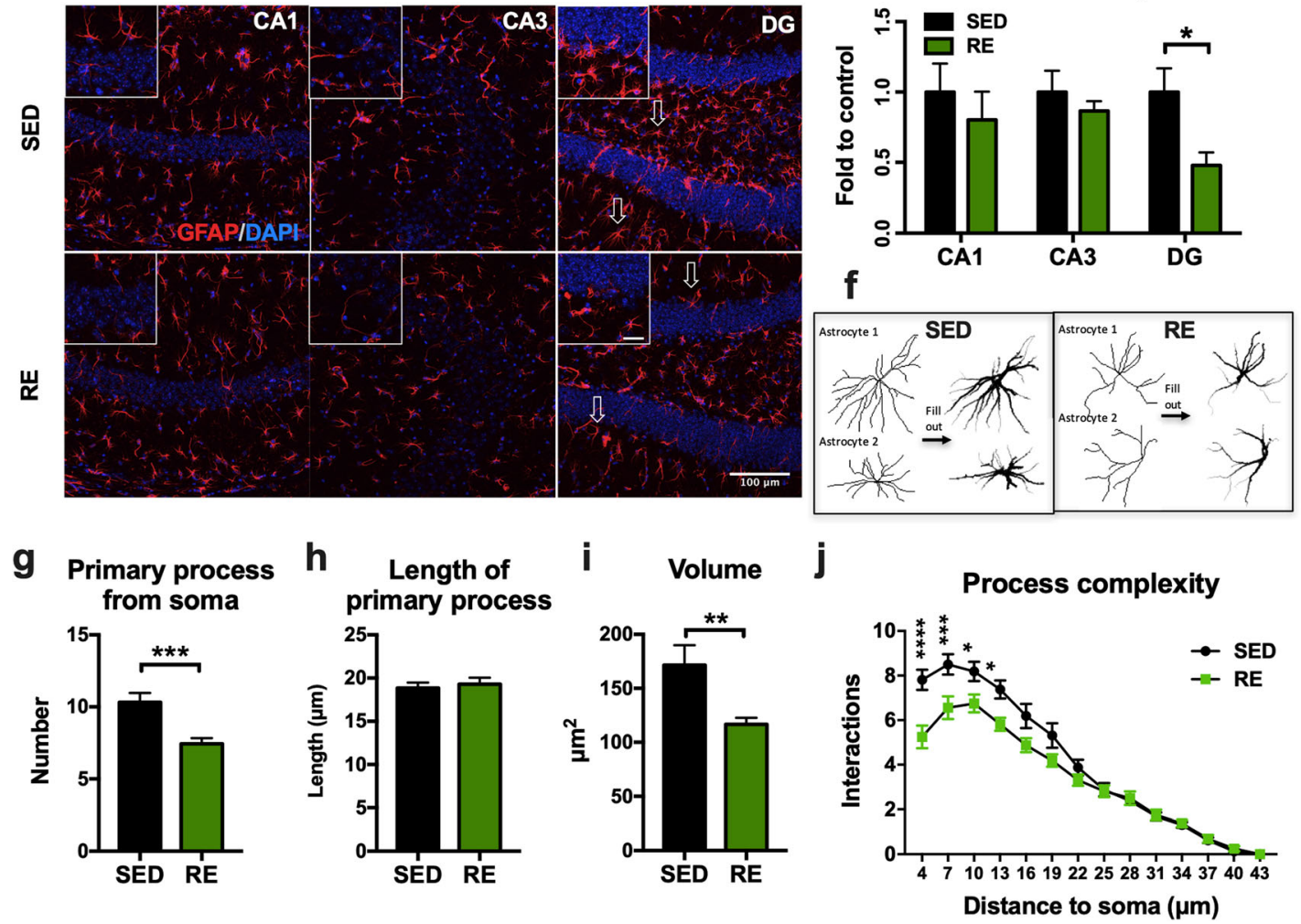

Fig. 6 (See legend on next page.) 
(See figure on previous page.)

Fig. 6 Effects of resistance exercise on microglial activation. a Representative confocal photographs taken with a $\times 20$ objective lens showing the Iba-1-positive microglia (green) and DAPI (blue) in the frontal cortex and different sub-regions of hippocampus. Scale bar: $100 \mu \mathrm{m}$ (for zoomed in images, scale bar $=20 \mu \mathrm{m}$ ). The number of microglia in each brain region was measured using ImageJ. b Cell count of Iba-1-positive microglia in the frontal cortex and different sub-regions of the hippocampus. c Cell body size of microglia in the frontal cortex and different subregions of the hippocampus. For each mouse, data represent the mean value of three brain slices. $\mathbf{d}$ Representative confocal photographs taken with a $\times 20$ objective lens showing the GFAP positive astrocyte (red) and DAPI (blue) in the different sub-regions of hippocampus. Scale bar: $100 \mu \mathrm{m}$. e Quantification of immunofluorescent intensity of the GFAP signal. $\mathbf{f}$ Morphological analysis of astrocytes, the representative picture showing the morphological feactures of astrocytes that were marked with arrows in picture $\mathbf{d}$. $\mathbf{g}-\mathbf{j}$ The result of morphological analysis of astrocyte in DG region. (unpaired Student's $t$ test, ${ }^{*} p<0.05,{ }^{* *} p<0.01,{ }^{* * *} p<0.001$, compared to sedentary mice. $n=5$ and 4 for IBA-1 and GFAP staining, respectively. Data presented as mean \pm SEM. SED sedentary, RE resistance exercise

$10 \mu \mathrm{m},-1.438 \pm 0.4609, p=0.0268$; radius $=13 \mu \mathrm{m}:-$ $1.563 \pm 0.4609, p=0.0107$; Fig. 6j).

Inflammatory factors and exercise-induced factors release following by resistance exercise training

Activated microglia and astrocytes secrete increased levels of pro-inflammatory cytokines, such as interleukin (IL) $-1 \beta$ and TNF- $\alpha$. Consistent with the reduction of activated microglia, we observed a significant decrease in the mRNA expression of TNF- $\alpha(-0.5605 \pm 0.1555, p=$ 0.0069; Fig. 7a) and IL-1 $\beta(-0.6519 \pm 0.1756, p=0.0059$; Fig. 7a) in the frontal cortex and liver respectively following resistance exercise. The anti-inflammatory mediator IL-10 was also increased in the hippocampus of the active compared to sedentary mice (1.078 \pm $0.4571, p=0.0461$; Fig. 7a). The downregulation of proinflammatory markers in the frontal cortex and liver, as well as the upregulation of anti-inflammatory markers in the hippocampus suggest a peripheral as well as central anti-inflammatory effect from resistant exercise. In the serum, we detected a significant lower protein level of IL- $1 \beta$ in exercised mice $(-2.339 \pm 0.8131, p=0.0183)$ but no differences were seen in the expression of IL-6 ( $p=0.1331$ ), IL-10, MCP-1, MIP-2, and TNF- $\alpha$.

The levels of a number of exercise-induced factors were changed following resistance exercise. IL-6 was increased in both the brain (FC: $1.535 \pm 0.6642, p=0.0496$ and hippocampus: $2.049 \pm 0.859, p=0.0442$ ) and the liver (1.145 $\pm 0.241, p=0.0014$, Fig. 7b). FGF-21 was also increased in the hippocampus $(4.461 \pm 1.899, p=0.0468$; Fig. 7b). PGC1- $\alpha$ was significantly increased in both the hippocampus $(0.5686 \pm 0.2238, p=0.0346)$ and the liver $(2.729 \pm 1.176, p=0.0489$; Fig. 7b).

\section{Changes in intracellular signal transduction proteins in the brain following resistance exercise}

The above findings suggest a protective role of resistance training against cognitive impairment, synaptic dysfunction, neuropathology, and neuroinflammation. We next investigated the intracellular mechanism associated with these changes. The phosphorylated Akt (ser 473) was significantly increased by resistance training (FC: $0.5592 \pm 0.1988, p=0.0184$; hippocampus: $1.074 \pm$
0.1991, $p=0.0003$; Fig. 8b, f), along with a significant increase of GSK-3 $\beta$ (Ser9) in the hippocampus (FC: $1.18 \pm 0.5526, p=0.0586$; hippocampus: $1.581 \pm 0.3045$, $p=0.0004$; Fig. 8b, f); while phosphorylated JNK (Thr183/ Tyr185) was significantly decreased following resistant training (FC: $-0.5258 \pm 0.1369, p=0.0033$; hippocampus: $-0.5126 \pm 0.1084, p=0.0008$; Fig. 8c, g). The protein levels of the regulators in Akt/GSK-3 $\beta$, MAPKs, and apoptotic pathway were not changed by resistance training in wildtype mice (Additional file 3a-c).

\section{Discussion}

Currently, there are no disease-modifying drugs available for Alzheimer's disease and available pharmacological and supportive therapy are aimed at slowing disease progression. Clinical studies suggest physical exercise improves cognitive function and reduces the risk of developing AD [20]. The majority of the relevant studies are focused on the benefits of aerobic exercise, with only relatively few studies addressing whether resistance exercise, especially on a short-term basis, can improve learning and memory. To the best of our knowledge, shortterm resistance exercise has not been previously shown to confer beneficial effects on cognitive function and neuropathology in 3xTg-AD mice and data from this study represents a novel finding.

Synaptic vesicle recycling and structural homeostasis are essential for maintaining normal cognitive function [19]. Neurotransmitters are released from synaptic nerve terminals via synaptic vesicle in response to a threshold action potential and the subsequent calcium influx. Therefore, functional improvement in the cognitive performance is likely to involve synaptic vesicle protein and synaptic structural protein changes. Indeed, we found that resistance exercise increased the expression of synaptotagmin-1 in the synaptosome fractions from the frontal cortex. Synaptotagmin-1 is a key calcium sensor for synaptic vesicle exocytosis, and have been implicated in synaptic vesicle endocytosis. Decrease in synaptotagmin-1 may lead to synaptic vesicle recycling disruption [21]. Synaptobrevin-1 was increased in the synaptosome fractions from hippocampal tissue after resistance exercise. This protein is essential for membrane 
a Pro- and anti-inflammatory cytokines

Frontal cortex

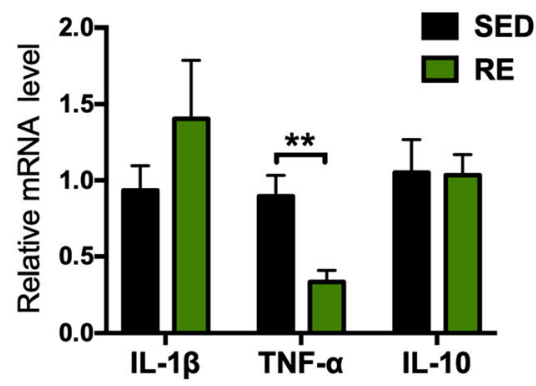

Hippocampus

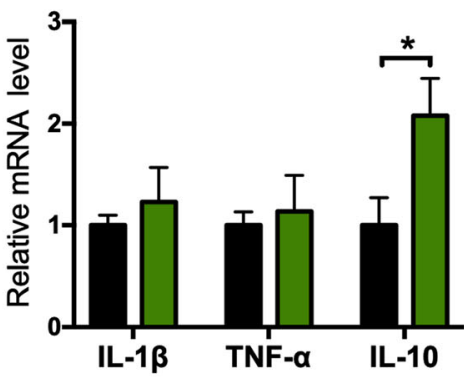

Liver

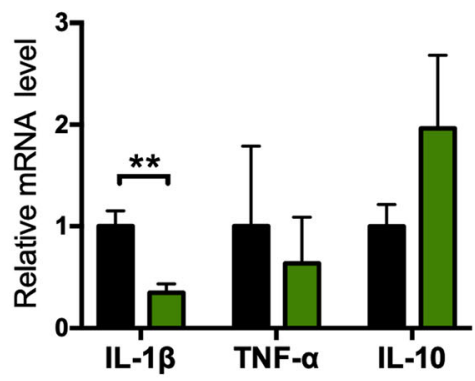

b

\section{Cytokines in serum}

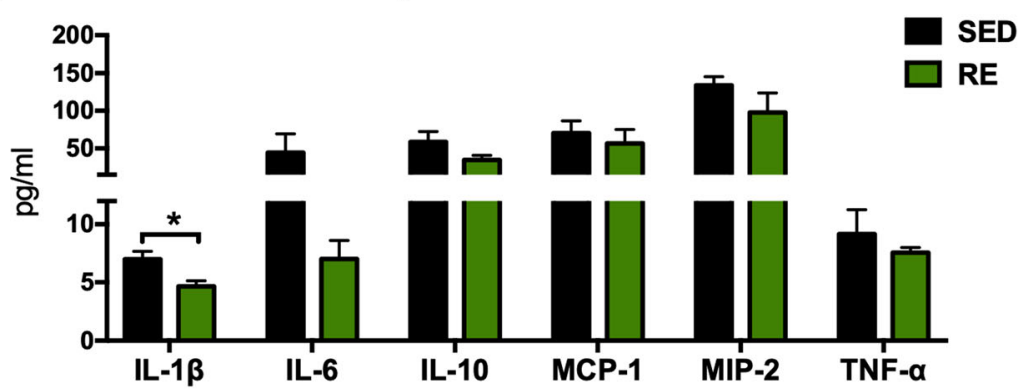

\section{Exercise-induced factors}

Frontal cortex

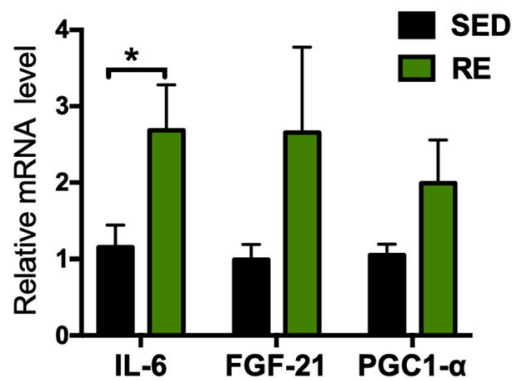

Hippocampus

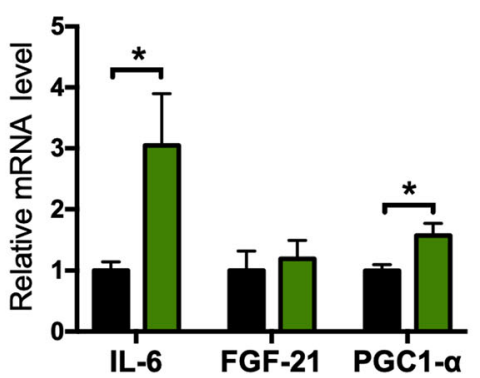

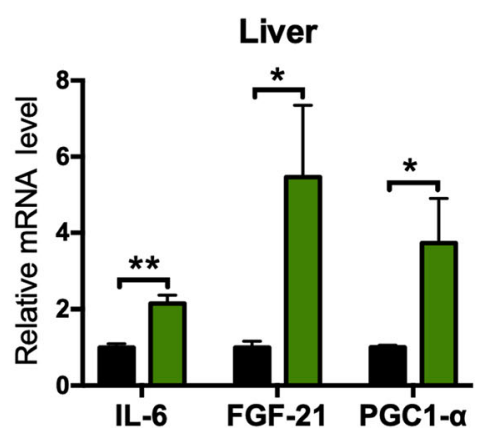

Fig. $\mathbf{7}$ Inflammatory and exercise induced factors following resistance exercise. a Relative mRNA levels for various inflammatory cytokines in the brain and liver. $\mathbf{b}$ Protein levels of various inflammatory cytokines in the serum. $\mathbf{c}$ Relative mRNA levels for exercise induced factors in the brain and liver (unpaired Student's $t$ test, ${ }^{*} p<0.05,{ }^{*} p<0.01$, compared to sedentary mice. $n=5$ for RT-PCR and 6 for Milliplex assay, data present as mean $\pm \mathrm{SEM})$. SED sedentary, $R E$ resistance exercise

fusion and decline in synaptobrevin-1 is reported to cause impaired synaptic development and induces slow neurodegeneration [22]. However, no difference was found in the levels of synapsin I or PSD 95. Thus, resistance exercise appeared to improve presynaptic vesicle recycling and synaptic transmission, rather than postsynaptic structure.

Translation of the overexpressed AD-related genes in the $3 \times \mathrm{Tg}$ mice is restricted mainly to the cerebral cortex and hippocampus, displayed as amyloid- $\beta$ plaques and tau pathology, both of which are associated with malfunctioning of synapses. Aerobic exercise has been shown to reduce $A \beta$ neurotoxicity [23] and tau pathogenesis [24] in the brain. Similar to these findings, we demonstrated a prominent reduction in amyloid deposits in the frontal cortex and hippocampus. Moreover, resistance training reduced the expression of hyperphosphorylated tau at the AT180 epitope. AT180 is a monoclonal antibody that specifically binds with tau at $\mathrm{Thr}$ 231 and is widely used to evaluate tau in tangles and paired helical filaments (PHFs) [25]. Combining with our result showing that resistance training decreased the expression of pan tau, these data indicated that the short-term resistance training reduced $\mathrm{A} \beta$ deposition, tau hypophosphorylation, and total tau burden in 3xTg mice. 


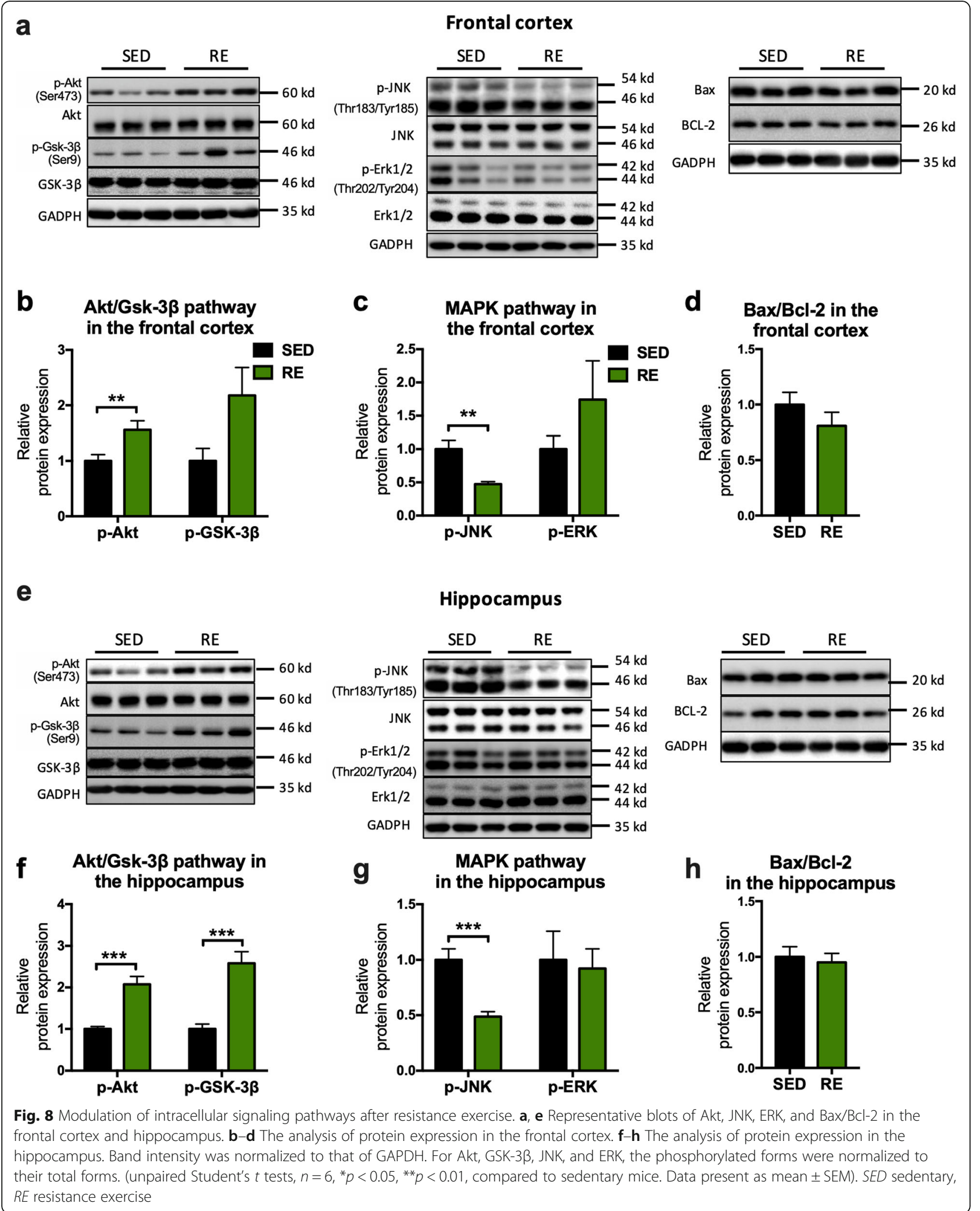


Mounting evidence suggests that AD probably results from neuroinflammatory response that are triggered by accumulating amyloid plaques and neurofibrillary tangles (NFTs) [26]. This inflammatory hypothesis is supported by findings from clinical studies and animal experiments showing that anti-inflammatory agents attenuated neuronal dysfunction in $\mathrm{AD}[27,28]$. Physical exercise confers broad anti-inflammatory effects in many neurodegenerative diseases, probably by affecting the secretion of inflammatory cytokines/adipokines via the muscle-adipose crosstalk $[29,30]$. It has been shown that exercise can improve the immune status of the brain, either by inhibiting the production of pro-inflammatory factors such as IL-1 $\beta$ [31], or by promoting the synthesis of anti-inflammatory factors such as IL-10 [32]. Interestingly, some studies demonstrate that the antiinflammatory effect of exercise in $\mathrm{AD}$ was attributed to the clearance of amyloid plaques [23], the plaques being a trigger of inflammation. Activated microglia and reactive astrocytes are identified as inflammatory pathology markers in AD [33]. In this study, we showed a decrease in microglial activation in the frontal cortex and hippocampus after resistance training, accompanied by a decrease in the activation of astrocytes in the DG region of the hippocampus. Activated microglia and astrocyte are the main source of pro-inflammatory cytokines in the brain. Consistent with this, there was also a decrease in mRNA expression of TNF- $\alpha$ in the frontal cortex and increased mRNA expression of IL-10 in the hippocampus. Previous studies has shown that levels of TNF- $\alpha$ is increased in the frontal cortex [34] but remained stable in the hippocampus in 3xTg mice [35]. The distinct changes of TNF- $\alpha$ levels in the fontal cortex compared to the hippocampus could be due to the differential transcription of different cytokines in different brain regions. IL-10 is one of the more important antiinflammatory cytokines. Signaling molecules including Akt and JNK has been shown to regulate the production of IL-10 [36], and the elevated expression of IL-10 in the hippocampus was consistent with our findings with the intracellular signaling pathways. However, the level of IL-10 remained unchanged in the fontal cortex, indicating that the molecular mechanisms regulating IL-10 production in the brain maybe region specific and are complex.

Systemic inflammation is well-established to contribute to the progression of $\mathrm{AD}$ and has been shown to predate the deposition of neurofibrillary tangles and amyloid plaques [37]. Adipocytes are important sources of peripheral inflammatory cytokines. Recent data demonstrated a direct anti-inflammatory effect of exercise by suppressing the production of several major proinflammatory cytokines including TNF- $\alpha$ and MCP-1 by adipocytes [38]. Similarly, we also identified a decreased
mRNA expression and protein level of IL-1 $\beta$ in the liver and serum, respectively in the active mice.

Interestingly, there was also an increase in the proinflammatory cytokine IL-6, in both the brain and liver of the active group. IL- 6 is considered different from IL$1 \beta$ and TNF- $\alpha$, in that the latter two are considered near-universal pro-inflammatory factors. IL-6, on the other hand, mediate multiple functions. IL- 6 participates in both trans-signaling and the classic signaling pathways during the inflammatory response and both pathways induce a complex range of responses, even in absence of inflammation. It is therefore argued that IL-6 plays a role in somatic efforts in maintenance, including resistance against inflammation, pathogen tolerance, and tissue repair [39]. More importantly, in our model, resistance exercise induced IL- 6 secretion is not accompanied by an elevation in IL- $1 \beta$ and TNF- $\alpha$. Rather, resistance exercise stimulated the production of the antiinflammatory cytokine IL-10. Through classic signaling, IL-6 upregulates lipolysis in viscera [40, 41], which might explain the significant body weight loss, attributed to mainly the reduction in adipose tissue, and the decrease in pro-inflammatory factors that were observed in the resistance exercise group. On balance, it could be argued that IL-6 release following resistance exercise is more anti-inflammatory than pro-inflammatory in its net effect.

Other than IL-6, we also evaluated the expression of two other exercise-induced cytokines, fibroblast growth factor 21 (FGF-21) and PGC-1 $\alpha$ [42]. FGF-21 is mainly produced in the liver and can permeate into CNS through the blood-brain barrier (BBB), binding with its essential co-receptors in the brain, thereby mediating neuroprotective effects [43]. The mRNA level of FGF-21 remained unchanged both in the frontal cortex and hippocampus, which is reasonable as FGF-21 is reported to be primarily generated in peripheral organs. However, there was a remarkable increase in expression of FGF-21 in the liver. Previous study showed that peripherally derived FGF-21 leaked into the injured brain and facilitate remyelination in a mouse model of lysophosphatidylcholine-induced demyelination [44]. In addition, subcutaneously injection of recombinant human FGF-21 improved cognitive function via attenuating insulin resistance and inflammatory response in an obese mouse model [45]. Whether elevated expression of FGF-21 following our resistant training also contributed to the benefits seen in the brain should be further determined.

PGC- $1 \alpha$ is a transcriptional co-activator that is preferentially expressed in high-energy requiring cells and is responsible for mediating oxidative metabolism, as well as mitochondrial biogenesis [46]. PGC- $1 \alpha$ has been shown to be dramatically upregulated following both 
aerobic and resistance exercise, contributing to exerciseinduced benefits to the CNS [47]. A recent review of the effects of PGC- $1 \alpha$ on AD pathophysiology suggest that PGC- $1 \alpha$ could improve oxidative stress, mitochondrial dysfunction, and insulin resistance, thereby preventing neuronal cell damage and ameliorating cognitive impairment in $\mathrm{AD}$ [48]. Moreover, Cheng et al. demonstrated a novel role of PGC- $1 \alpha$ in the dendritic spine formation and maintenance in the hippocampus [49]. On the other hand, irisin, the secreted form of fibronectin type III domain containing protein 5 (FNDC5) (a PGC- $1 \alpha$ dependent membrane-bound protein), has been demonstrated to suppress the secretion of TNF- $\alpha$ and MCP-1 by adipocytes [38], suggesting a direct anti-inflammatory impact of PGC-1 $\alpha$. The enhanced level of PGC-1 $\alpha$ after resistance exercise we observed in the liver and hippocampus might contribute to the reduced inflammation and improved synaptic transmission, respectively. On the other hand, the SIRT1-PGC1- $\alpha$ axis has been shown to be associated with exercise-induced mitochondrial biogenesis, which could counteract excessive mitochondrial fission and degradation in neurodegenerative diseases [50]. Moreover, it is increasingly that mitochondrial dysfunction and neuroinflammation are interdependent lesions in $\mathrm{AD}$. The direct or indirect manipulation of mitochondria through interfering with bioenergetic pathways could reduce neuroinflammation in $\mathrm{AD}$ [51]. Therefore, the correlation of elevated mRNA level of PGC1- $\alpha$ with mitochondrial biogenesis, and their relationship with neuroinflammation should be further studied using the current model.

We proceeded to investigate possible signaling pathways involved in the resistance exercise-induced beneficial effects. The activity of Akt/GSK3 $\beta$ and mitogen-activated protein kinases (MAPKs) were evaluated as the former is pivotal to cellular proliferation, growth, and survival in response to extracellular stimuli [52] and the latter playing a key part in regulating intracellular adaptions to various extracellular stimulation such as hyperosmosis, oxidative stress, and pro-inflammatory factors [52]. A $\beta$ oligomer inhibits the PI3K/AKT pathway, and pharmacological activation of Akt activity reduces synaptic deficits and cognitive impairment in both 5XFAD and $A \beta$-induced AD mice [53]. Activities of GSK-3 $\beta$, the downstream factor of Akt, is closely linked with tau hyperphosphorylation and memory dysfunction that triggered by soluble form of Amyloid- $\beta$ [54]. We showed a significant activation of Akt (Ser 473) and GSK-3ß (Ser 9) in both the frontal cortex and hippocampus. The combining data from ours and Ali et al.'s study suggest that resistance exercise might reduce $A \beta$ deposits and tau pathology by increasing the activity of the Akt/GSK-3 $\beta$ signal pathway.

The MAPK signal pathways have been shown to be activated in vulnerable brain regions of $\mathrm{AD}$ patients and have been proposed as therapeutic targets for AD [55]. MAPK contains the extracellular signal-regulated kinase $1 / 2($ ERK1/2) pathways that are essential for cell survival and the c-Jun N-terminal kinase (JNK) pathways that are vital for mediating inflammatory response. Previous studies showed that the inhibition of JNK activation attenuated amyloid plaque, tau hyperphosphorylation and cognitive deficiency in APPswe/PS1dE9 mice [56]. Although it has been shown that activation of ERK1/2 exacerbated the $\mathrm{AD}$ phenotype in $5 \mathrm{X}$ familial mice [57], few studies demonstrated the direct effect of inhibition of ERK activity in the AD phenotype. In the present study, inhibition of JNK (Thr183/Tyr185) by resistance exercise was demonstrated in both the frontal cortex and hippocampus. However, no effect of exercise training on the activity of ERK1/2 or its downstream molecules, Bcl-2 and Bax, two well-known markers of cell death were identified. Hence, resistance exercise might protect the AD brain from neuroinflammation and subsequent neuropathology by inhibition of the JNK pathway.

\section{Conclusions}

To conclude, the stress-free short-term resistance exercise training confers a range of beneficial effects on the brains of 3xTg-AD mice including increasing synaptic structural proteins, reducing amyloid deposit and tau pathology, as well as attenuating neuroinflammation that culminate into improving cognitive performance. The mediation of these effects is likely to involve the Akt/ GSK-3 $\beta$ and JNK signal pathway molecules that may represent potential therapeutic targets for AD.

\section{Supplementary information}

Supplementary information accompanies this paper at https://doi.org/10 1186/s12974-019-1653-7.

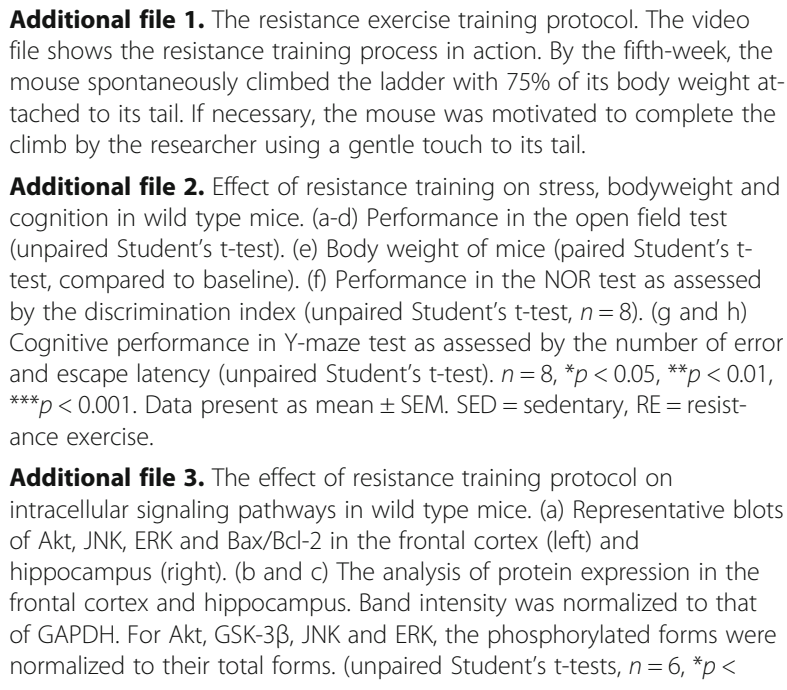

Additional file 1. The resistance exercise training protocol. The video file shows the resistance training process in action. By the fifth-week, the mouse spontaneously climbed the ladder with $75 \%$ of its body weight at tached to its tail. If necessary, the mouse was motivated to complete the climb by the researcher using a gentle touch to its tail.

Additional file 2. Effect of resistance training on stress, bodyweight and cognition in wild type mice. (a-d) Performance in the open field test (unpaired Student's t-test). (e) Body weight of mice (paired Student's ttest, compared to baseline). (f) Performance in the NOR test as assessed by the discrimination index (unpaired Student's t-test, $n=8$ ). ( $\mathrm{g}$ and $\mathrm{h}$ ) Cognitive performance in $Y$-maze test as assessed by the number of error and escape latency (unpaired Student's t-test). $n=8,{ }^{*} p<0.05,{ }^{* *} p<0.01$, ${ }^{* * *} p<0.001$. Data present as mean \pm SEM. SED $=$ sedentary, RE $=$ resistance exercise.

Additional file 3. The effect of resistance training protocol on intracellular signaling pathways in wild type mice. (a) Representative blots of Akt, JNK, ERK and Bax/Bcl-2 in the frontal cortex (left) and hippocampus (right). (b and c) The analysis of protein expression in the frontal cortex and hippocampus. Band intensity was normalized to that of GAPDH. For Akt, GSK-3 $\beta$, JNK and ERK, the phosphorylated forms were normalized to their total forms. (unpaired Student's t-tests, $n=6,{ }^{*} p<$ 
$0.05{ }^{* *} p<0.01$, compared to sedentary mice. Data present as mean \pm $\mathrm{SEM}) . \mathrm{SED}=$ sedentary, $\mathrm{RE}=$ resistance exercise

\section{Abbreviations}

AD: Alzheimer's disease; CNS: Central nervous system; ERK: Extracellular signal-regulated kinases; FGF: Fibroblast growth factor;

GAPDH: Glyceraldehyde 3-phosphate dehydrogenase; IL: Interleukin; JNK: CJun N-terminal kinases; NFTs: Neurofibrillary tangles; NOR: Novel object recognition; PGC1-a: Peroxisome proliferator-activated receptor gamma coactivator 1-alpha; PSD95: Postsynaptic density 95; RE: Resistance exercise; SED: Sedentary; Tg: Transgenic; TNF: Tumor necrosis factor; WT: Wild type

\section{Acknowledgments}

We thank Dr. PANG, Pei Shin and Dr. GUO, Jing from Faculty Core Facility, the University of Hong Kong for the excellent technical assistance and helpful discussions regarding confocal imaging.

\section{Authors' contributions}

YL performed the experiments and drafted the manuscript. JMTC and TY analyzed the number of microglia and amyloid deposits. $Y L, Y Z$, and $Y C$ analyzed the data of behavior, RT-PCR, and Western Blot. JMTC, RCCC, and GTCW contributed to the experimental design and manuscript editing and revision. All authors read and approved the final manuscript.

\section{Funding}

This project was supported by HKU Seed Fund for Basic Research (201511159317 and 201611159054).

\section{Availability of data and materials}

The datasets during and/or analyzed during the current study available from the corresponding author on reasonable request.

\section{Ethics approval}

The use of animals was approved by the Department of Health, Hong Kong and the Committee on the Use of Live Animals in Teaching and Research (CULATR), The University of Hong Kong. The approval number was 4192-16.

\section{Consent for publication}

Not applicable.

\section{Competing interests}

The authors declare that they have no competing interests.

\section{Author details}

'Department of Anaesthesiology, LKS Faculty of Medicine, The University of Hong Kong, Room K424, Queen Mary Hospital, Pokfulam, Hong Kong, SAR, China. ${ }^{2}$ Laboratory of Neurodegenerative Diseases, LKS Faculty of MedicineSchool of Biomedical Sciences, The University of Hong Kong, Hong Kong, SAR, China. ${ }^{3}$ State Key Laboratory of Brain and Cognitive Sciences, The University of Hong Kong, L4-49, Laboratory Block, Pokfulam, Hong Kong, SAR, China.

\section{Received: 15 August 2019 Accepted: 20 November 2019}

\section{Published online: 03 January 2020}

\section{References}

1. Mirra SS, Heyman A, McKeel D, Sumi S, Crain BJ, Brownlee L, et al. The Consortium to Establish a Registry for Alzheimer's Disease (CERAD) Part II. Standardization of the neuropathologic assessment of Alzheimer's disease. Neurology. 1991:41:479.

2. Heneka MT, Carson MJ, El Khoury J, Landreth GE, Brosseron F, Feinstein DL, et al. Neuroinflammation in Alzheimer's disease. The Lancet Neurol. 2015;14 388-405.

3. Walker D, Kim S, McGeer P. Complement and cytokine gene expression in cultured microglia derived from postmortem human brains. J Neurosci Res. 1995:40:478-93.

4. Hopperton KE, Mohammad D, Trepanier MO, Giuliano V, Bazinet RP. Markers of microglia in post-mortem brain samples from patients with Alzheimer's disease: a systematic review. Mol Psychiatry. 2018;23:177-98.
5. Streit WJ, Mrak RE, Griffin WST. Microglia and neuroinflammation: a pathological perspective. J Neuroinflammation. 2004;1:14.

6. Calsolaro V, Edison P. Neuroinflammation in Alzheimer's disease: current evidence and future directions. Alzheimers Dement. 2016;12:719-32.

7. Vlad SC, Miller DR, Kowall NW, Felson DT. Protective effects of NSAIDs on the development of Alzheimer disease. Neurology. 2008;70:1672-7.

8. Azam F, Alabdullah NH, Ehmedat HM, Abulifa AR, Taban I, Upadhyayula S. NSAIDs as potential treatment option for preventing amyloid beta toxicity in Alzheimer's disease: an investigation by docking, molecular dynamics, and DFT studies. J Biomol Struct Dyn. 2018;36:2099-117.

9. Radak Z, Hart N, Sarga L, Koltai E, Atalay M, Ohno H, et al. Exercise plays a preventive role against Alzheimer's disease. J Alzheimers Dis. 2010;20:777-83.

10. Hawley JA, Hargreaves M, Joyner MJ, Zierath JR. Integrative biology of exercise. Cell. 2014;159:738-49.

11. Brown B, Peiffer J, Martins R. Multiple effects of physical activity on molecular and cognitive signs of brain aging: can exercise slow neurodegeneration and delay Alzheimer's disease? Mol Psychiatry. 2013;18:864.

12. Geda YE, Roberts RO, Knopman DS, Christianson TJ, Pankratz VS, Ivnik RJ, et al. Physical exercise, aging, and mild cognitive impairment: a populationbased study. Arch Neurol. 2010;67:80-6.

13. Adlard PA, Perreau VM, Pop V, Cotman CW. Voluntary exercise decreases amyloid load in a transgenic model of Alzheimer's disease. J Neurosci. 2005;25:4217-21.

14. Svensson M, Lexell J, Deierborg T. Effects of physical exercise on neuroinflammation, neuroplasticity, neurodegeneration, and behavior: what we can learn from animal models in clinical settings. Neurorehabil Neural Repair. 2015;29:577-89.

15. Kim HJ, So B, Choi M, Kang D, Song W. Resistance exercise training increases the expression of irisin concomitant with improvement of muscle function in aging mice and humans. Exp Gerontol. 2015:70:11-7.

16. Huang C, Chu JM, Liu Y, Chang RC, Wong GT. Varenicline reduces DNA damage, tau mislocalization and post surgical cognitive impairment in aged mice. Neuropharmacology. 2018;143:217-27.

17. Tavares G, Martins M, Correia JS, Sardinha VM, Guerra-Gomes S, das Neves SP, et al. Employing an open-source tool to assess astrocyte tridimensional structure. Brain Struct Funct. 2017;222:1989-99.

18. Zheng Y, Manson JE, Yuan C, Liang MH, Grodstein F, Stampfer MJ, et al. Associations of weight gain from early to middle adulthood with major health outcomes later in life. Jama. 2017;318:255-69.

19. Tonnies E, Trushina E. Oxidative stress, synaptic dysfunction, and Alzheimer's disease. J Alzheimers Dis. 2017:57:1105-21.

20. Frederiksen KS, Gjerum L, Waldemar G, Hasselbalch SG. Effects of physical exercise on Alzheimer's disease biomarkers: a systematic review of intervention studies. J Alzheimers Dis. 2018;61:359-72.

21. Li YC, Chanaday NL, Xu W, Kavalali ET. Synaptotagmin-1- and synaptotagmin-7-dependent fusion mechanisms target synaptic vesicles to kinetically distinct endocytic pathways. Neuron. 2017:93:616-31. e3

22. Haberman A, Williamson WR, Epstein D, Wang D, Rina S, Meinertzhagen IA, et al. The synaptic vesicle SNARE neuronal Synaptobrevin promotes endolysosomal degradation and prevents neurodegeneration. J Cell Biol. 2012;196:261-76.

23. Prado Lima MG, Schimidt HL, Garcia A, Dare LR, Carpes FP, Izquierdo I, et al. Environmental enrichment and exercise are better than social enrichment to reduce memory deficits in amyloid beta neurotoxicity. Proc Natl Acad Sci U S A. 2018;115:E2403-e9.

24. Gratuze M, Julien J, Morin F, Marette A, Planel E. Differential effects of voluntary treadmill exercise and caloric restriction on tau pathogenesis in a mouse model of Alzheimer's disease-like tau pathology fed with Western diet. Prog Neuro-Psychopharmacol Biol Psychiatry. 2017;79:452-61.

25. Amniai L, Lippens G, Landrieu I. Characterization of the AT180 epitope of phosphorylated tau protein by a combined nuclear magnetic resonance and fluorescence spectroscopy approach. Biochem Biophys Res Commun. 2011;412:743-6.

26. McGeer PL, McGeer EG. The amyloid cascade-inflammatory hypothesis of Alzheimer disease: implications for therapy. Acta Neuropathol. 2013;126:479-97.

27. Moussa C, Hebron M, Huang X, Ahn J, Rissman RA, Aisen PS, et al. Resveratrol regulates neuro-inflammation and induces adaptive immunity in Alzheimer's disease. J Neuroinflammation. 2017;14:1.

28. Daniels MJ, Rivers-Auty J, Schilling T, Spencer NG, Watremez W, Fasolino V, et al. Fenamate NSAIDs inhibit the NLRP3 inflammasome and protect against Alzheimer's disease in rodent models. Nat Commun. 2016;7:12504. 
29. Stranahan AM, Martin B, Maudsley S. Anti-inflammatory effects of physical activity in relationship to improved cognitive status in humans and mouse models of Alzheimer's disease. Curr Alzheimer Res. 2012;9:86-92.

30. Majdinasab N, Motl RW, Mokhtarzade M, Zimmer P, Ranjbar R, Keytsman C, et al. Acute responses of cytokines and adipokines to aerobic exercise in relapsing vs. remitting women with multiple sclerosis. Complement Ther Clin Pract. 2018;31:295-301.

31. Ryan SM, Kelly AM. Exercise as a pro-cognitive, pro-neurogenic and antiinflammatory intervention in transgenic mouse models of Alzheimer's disease. Ageing Res Rev. 2016;27:77-92.

32. Gomes da Silva S, Simoes PS, Mortara RA, Scorza FA, Cavalheiro EA, da Graca N-MM, et al. Exercise-induced hippocampal anti-inflammatory response in aged rats. J Neuroinflammation. 2013;10:61.

33. Taipa R, Ferreira V, Brochado P, Robinson A, Reis I, Marques F, et al. Inflammatory pathology markers (activated microglia and reactive astrocytes) in early and late onset Alzheimer disease: a post mortem study. Neuropathol Appl Neurobiol. 2018:44:298-313.

34. Culpan D, Cram D, Chalmers K, Cornish A, Palmer L, Palmer J, et al. TNFRassociated factor-2 (TRAF-2) in Alzheimer's disease. Neurobiol Aging. 2009; 30:1052-60.

35. Janelsins MC, Mastrangelo MA, Oddo S, LaFerla FM, Federoff HJ, Bowers WJ. Early correlation of microglial activation with enhanced tumor necrosis factor-alpha and monocyte chemoattractant protein-1 expression specifically within the entorhinal cortex of triple transgenic Alzheimer's disease mice. J Neuroinflammation. 2005;2:23.

36. Lobo-Silva D, Carriche GM, Castro AG, Roque S, Saraiva M. Balancing the immune response in the brain: IL-10 and its regulation. J Neuroinflammation. 2016;13:297.

37. Holmes C. Review: systemic inflammation and Alzheimer's disease. Neuropathol Appl Neurobiol. 2013;39:51-68.

38. Mazur-Bialy Al, Bilski J, Pochec E, Brzozowski T. New insight into the direct anti-inflammatory activity of a myokine irisin against proinflammatory activation of adipocytes. Implication for exercise in obesity. J Physiol Pharmacol. 2017;68:243-51.

39. Del Giudice M, Gangestad SW. Rethinking IL-6 and CRP: why they are more than inflammatory biomarkers, and why it matters. Brain Behav Immun. 2018;70:61-75

40. Ma Y, Gao M, Sun H, Liu D. Interleukin-6 gene transfer reverses body weight gain and fatty liver in obese mice. Biochim Biophys Acta. 1852; 2015:1001-11.

41. Grisouard J, Bouillet E, Timper K, Radimerski T, Dembinski K, Frey DM, et al. Both inflammatory and classical lipolytic pathways are involved in lipopolysaccharide-induced lipolysis in human adipocytes. Innate Immun. 2012;18:25-34.

42. Liu Y, Yan T, Chu JM, Chen Y, Dunnett S, Ho YS, et al. The beneficial effects of physical exercise in the brain and related pathophysiological mechanisms in neurodegenerative diseases. Lab Investig. 2019;99(7):943-57.

43. Fisher FM, Maratos-Flier E. Understanding the physiology of FGF21. Annu Rev Physiol. 2016;78:223-41.

44. Kuroda M, Muramatsu R, Maedera N, Koyama Y, Hamaguchi M, Fujimura H, et al. Peripherally derived FGF21 promotes remyelination in the central nervous system. J Clin Invest. 2017;127:3496-509.

45. Wang Q, Yuan J, Yu Z, Lin L, Jiang Y, Cao Z, et al. FGF21 attenuates high-fat diet-induced cognitive impairment via metabolic regulation and antiinflammation of obese mice. Mol Neurobiol. 2018;55:4702-17.

46. Andersson U, Scarpulla RC. Pgc-1-related coactivator, a novel, seruminducible coactivator of nuclear respiratory factor 1-dependent transcription in mammalian cells. Mol Cell Biol. 2001;21:3738-49.

47. Ji LL, Dickman JR, Kang C, Koenig R. Exercise-induced hormesis may help healthy aging. Dose-Response. 2010;8:73-9.

48. Sweeney G, Song J. The association between PGC-1alpha and Alzheimer's disease. Anat Cell Biol. 2016;49:1-6.

49. Cheng A, Wan R, Yang JL, Kamimura N, Son TG, Ouyang $X$, et al. Involvement of PGC-1alpha in the formation and maintenance of neurona dendritic spines. Nat Commun. 2012;3:1250.

50. Marques-Aleixo I, Oliveira PJ, Moreira PI, Magalhaes J, Ascensao A. Physical exercise as a possible strategy for brain protection: evidence from mitochondrial-mediated mechanisms. Prog Neurobiol. 2012;99:149-62.

51. Wilkins HM, Swerdlow RH. Relationships between mitochondria and neuroinflammation: implications for Alzheimer's disease. Curr Top Med Chem. 2016;16:849-57.
52. Chu J, Lauretti E, Pratico D. Caspase-3-dependent cleavage of Akt modulates tau phosphorylation via GSK3beta kinase: implications for Alzheimer's disease. Mol Psychiatry. 2017;22:1002-8.

53. Yi JH, Baek SJ, Heo S, Park HJ, Kwon H, Lee S, et al. Direct pharmacological Akt activation rescues Alzheimer's disease like memory impairments and aberrant synaptic plasticity. Neuropharmacology. 2018;128:282-92.

54. Tokutake T, Kasuga K, Yajima R, Sekine Y, Tezuka T, Nishizawa M, et al. Hyperphosphorylation of tau induced by naturally secreted amyloid-beta at nanomolar concentrations is modulated by insulin-dependent Akt-GSK3beta signaling pathway. J Biol Chem. 2012;287:35222-33.

55. Zhu X, Lee HG, Raina AK, Perry G, Smith MA. The role of mitogen-activated protein kinase pathways in Alzheimer's disease. Neurosignals. 2002;11:270-81.

56. Zhou Q, Wang M, Du Y, Zhang W, Bai M, Zhang Z, et al. Inhibition of c-Jun $\mathrm{N}$-terminal kinase activation reverses Alzheimer disease phenotypes in APPswe/PS1dE9 mice. Ann Neurol. 2015:77:637-54.

57. Stygelbout V, Leroy K, Pouillon V, Ando K, D'Amico E, Jia Y, et al. Inositol trisphosphate 3-kinase $B$ is increased in human Alzheimer brain and exacerbates mouse Alzheimer pathology. Brain. 2014;137:537-52.

\section{Publisher's Note}

Springer Nature remains neutral with regard to jurisdictional claims in published maps and institutional affiliations.

\section{Ready to submit your research? Choose BMC and benefit from:}

- fast, convenient online submission

- thorough peer review by experienced researchers in your field

- rapid publication on acceptance

- support for research data, including large and complex data types

- gold Open Access which fosters wider collaboration and increased citations

- maximum visibility for your research: over $100 \mathrm{M}$ website views per year

At BMC, research is always in progress.

Learn more biomedcentral.com/submissions 